\title{
Minimal eventually positive realizations of externally positive systems
}

\author{
Claudio Altafini ${ }^{\text {a }}$ \\ ${ }^{a}$ Division of Automatic Control, Dept. of Electrical Engineering, Linköping University, SE-58183, Sweden.
}

\begin{abstract}
It is a well-know fact that externally positive linear systems may fail to have a minimal positive realization. In order to investigate these cases, we introduce the notion of minimal eventually positive realization, for which the state update matrix becomes positive after a certain power. Eventually positive realizations capture the idea that in the impulse response of an externally positive system the state of a minimal realization may fail to be positive, but only transiently. As a consequence, we show that in discrete-time it is possible to use downsampling to obtain minimal positive realizations matching decimated sequences of Markov coefficients of the impulse response. In continuous-time, instead, if the sampling time is chosen sufficiently long, a minimal eventually positive realization leads always to a sampled realization which is minimal and positive.
\end{abstract}

Key words: positive linear systems; minimal realization; eventually positive matrices; Perron-Frobenius theorem.

\section{Introduction}

The positive realization problem for an externally (i.e., inputoutput) positive linear system consists in finding a state space representation which is itself positive, i.e., a triple $\{A, b, c\}$ for which $A, b$ and $c$ are nonnegative. The problem has been investigated for several decades, see Benvenuti \& Farina (2004); Farina \& Rinaldi (2000) for an introduction and a survey of the main results.

Unlike existence, which is well-characterized (Ohta et al. , 1984; Maeda \& Kodama, 1981; Farina \& Benvenuti, 1995; Anderson et al. , 1996), the problem of constructing positive realizations of minimal order is a difficult one, far from being completely solved. The positivity constraints, in fact, imply that not all externally positive systems admit a realization which is both minimal and positive, i.e., which is simultaneously positive, controllable and observable. There is by now a consistent literature on the topic, notably dealing with conditions on the order of the achievable realizations (Hadjicostis, 1999; Nagy \& Matolcsi, 2003), and developing algorithms to construct positive realizations in special cases (Benvenuti et al. , 2000; Benvenuti, 2013; Canto et al. , 2007; Kim, 2012; Nagy \& Matolcsi, 2005). However, systematic procedures for obtaining minimal positive realizations are in general unknown.

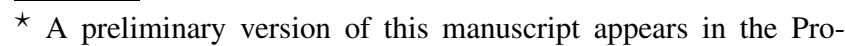
ceedings of the 54th IEEE Conference on Decision and Control, December 2015.
}

Email address: claudio.altafini@liu.se (Claudio Altafini).
Rather than contributing to this search, the scope of this paper is to investigate the structure of the minimal realization of externally positive systems, and to suggest a class of minimal realizations capturing the gap between external and internal positivity. The starting point of our approach is the observation that the fundamental mathematical principle behind positivity is the Perron-Frobenius theorem. In essence, existence of a positive realization is associated to existence of a polyhedral cone which is $A$-invariant (Ohta et al. , 1984; Anderson et al. , 1996). Such cone contains the positive eigenvector associated to the dominant eigenvalue, and at least when dominance is strict and the input is vanishing, the evolution of the linear system tends to become aligned with that eigenspace. If we relax the assumption of positivity of $A$ while maintaining the condition that the eigenvector must be contained in $\mathbb{R}_{+}^{n}$, then we still have that the free evolution of the state of a minimal realization becomes positive after a transient. Matrices $A$ having both left and right dominant eigenvector in $\mathbb{R}_{+}^{n}$ form a special class of matrices called eventually positive, see Altafini \& Lini (2015); Noutsos (2006). While these matrices can have negative entries (hence they do not correspond to positive realizations), they have the property that after a certain power they become positive matrices. Therefore in discrete-time this property naturally leads to free evolutions of the state variables that become nonnegative after a certain number of steps. If in addition the reachable cone associated to the realization is contained in $\mathbb{R}_{+}^{n}$, then the entire state vector must become positive after a transient. Notice that our approach is qualitatively different from Guidorzi (2014), where relaxing the positivity of $A$ may lead to state trajectories which never become positive when initialized outside the reachable cone. 
Formalizing our argument, we shown in the paper that a minimal realization in which $A$ is eventually positive and $b$ (resp. $c$ ) belongs to the corresponding $A$-invariant cone (resp. dual cone) is guaranteed to be externally positive. As for the converse, we provide constructive procedures to obtain a minimal eventually positive realization from a given externally positive system. While we do not have an explicit proof that every externally positive system admits a minimal realization of this type, it is tempting to conjecture that indeed it is so, at least in the case of a simple strictly dominant pole.

With respect to a preliminary version of this manuscript appearing as a conference paper (Altafini, 2015), the constructive procedure proposed here (Algorithm 1) is more general, and recovers the result of Altafini (2015) as a special case (Algorithm 2). Such special case is instrumental to show that a consequence of the existence of a minimal eventually positive realization is that the sequence of Markov parameters that compose the impulse response has decimated subsequences for which a minimal positive realization exists, and can be found downsampling the eventually positive realization. The number of steps it takes for $A$ to become positive gives a lower bound on the sought decimation factor. In continuous-time, instead, provided the sampling time is chosen sufficiently high, the sampled system obtained from a minimal eventually positive realization is itself positive and minimal. Also in this case (which is not treated in Altafini (2015)), once an eventually positive realization is available, a lower bound on the sampling time leading to a minimal positive sampled realization is known. These results on sampled/downsampled systems can be interpreted as a dual of the usual Nyquist-Shannon theorem: instead of seeking for a sampling frequency sufficiently high so as to preserve all interesting frequencies of the system, if one selects a sampling frequency enough low it is possible to achieve an internal minimal representation of the system which remains positive, because it disregards the high frequency content. In externally positive systems with strict dominance of the real eigenvalue, as we consider here, these frequencies are associated to non-dominant modes, hence they are necessarily transient. Therefore the nonpositive entries of our eventually positive realizations and the violations of positivity in the state vectors they induce must necessarily be associated to the non-dominant modes.

The rest of this paper is organized as follows: Section 2 recalls the basic linear algebraic notions associated to eventually positive matrices, which are then used in Section 3 to introduce eventually positive realizations in discrete-time. Constructive procedures are also given in this Section, while the downsampling of eventually positive realizations is studied in Section 4. In Section 5 continuous-time eventually positive realizations are investigated and analogous results are obtained.

\section{Linear algebra background}

For a matrix $A=\left(a_{i j}\right) \in \mathbb{R}^{n \times n}$, in this paper $A \geq 0$ means $a_{i j} \geq 0$ for any $i, j \in 1, \ldots, n$, and $A \neq 0$, while $A>0$ means $a_{i j}>0$ for all $i, j=1, \ldots, n$. The matrix $A$ is nonnegative (resp. positive) if $A \geq 0$ (resp. $A>0$ ). This notation is used also for vectors. The spectrum of $A$ is denoted $\operatorname{sp}(A)=\left\{\lambda_{1}(A), \ldots, \lambda_{n}(A)\right\}$, where $\lambda_{i}(A), i=1, \ldots, n$, are the eigenvalues of $A$. The spectral radius of $A, \rho(A)$, is the smallest real positive number such that $\rho(A) \geq\left|\lambda_{i}(A)\right|$, $\forall i=1, \ldots, n$.

\subsection{Eventually positive matrices}

Definition 1 A matrix $A \in \mathbb{R}^{n \times n}$ has the strong PerronFrobenius property if $\rho(A)$ is a simple positive eigenvalue of $A$ s.t. $\rho(A)>|\lambda|$ for every $\lambda \in \operatorname{sp}(A), \lambda \neq \rho(A)$, and $v$, the right eigenvector relative to $\rho(A)$, is positive.

Denote $\mathscr{P} \mathscr{F}_{n}$ the set of matrices in $\mathbb{R}^{n \times n}$ that possess the strong Perron-Frobenius property. These properties are associated to a class of matrices called eventually positive (Friedland, 1978; Johnson \& Tarazaga, 2004; Noutsos, 2006; Elhashash \& Szyld, 2008), class that is strictly bigger than that of positive matrices, in the sense that the matrices can contain negative entries.

Definition 2 A real square matrix $A$ is said to be eventually positive if $\exists \eta_{o} \in \mathbb{N}$ such that $A^{\eta}>0$ for all $\eta \geq \eta_{o}$.

The smallest integer $\eta_{o}$ of Definition 2 is called the power index of $A$. Following Olesky et al. (2009), eventually positive matrices will be denoted $A \stackrel{\vee}{>} 0$. For eventually positive matrices a necessary and sufficient condition for the fulfillment of the strong Perron-Frobenius property is available.

Theorem 1 (Noutsos (2006), Theorem 2.2) For $A \in \mathbb{R}^{n \times n}$ the following are equivalent:

(1) Both $A, A^{T} \in \mathscr{P}_{\mathscr{F}_{n}}$;

(2) $A \stackrel{\vee}{>} 0$;

(3) $A^{T} \stackrel{\vee}{>} 0$.

A matrix $A$ is said exponentially positive if $e^{A t}=\sum_{k=0}^{\infty} \frac{A^{k} t^{k}}{k !}>$ $0 \forall t \geq 0$, and $A$ is exponentially positive if and only if $A$ is Metzler, i.e., $a_{i j} \geq 0 \forall i \neq j$ (Noutsos \& Tsatsomeros, 2008; Ohta et al. , 1984).

Definition 3 A matrix $A \in \mathbb{R}^{n \times n}$ is said eventually exponentially positive if $\exists t_{o} \in[0, \infty)$ such that $e^{A t}>0 \forall t \geq t_{o}$.

We denote the smallest such $t_{o}$ the exponential index of $A$. The relationship between eventual positivity and eventual exponential positivity is given in Noutsos \& Tsatsomeros (2008) 
Theorem 2 (Noutsos \& Tsatsomeros (2008), Theorem 3.3) Given $A \in \mathbb{R}^{n \times n}$, A is eventually exponentially positive if and only if $\exists d \geq 0$ such that $A+d I \stackrel{\vee}{>} 0$.

\subsection{Invariant cones and eventually positive matrices}

A set $\mathscr{K} \subset \mathbb{R}^{n}$ is called a convex cone if $\alpha_{1} x_{1}+\alpha_{2} x_{2} \in$ $\mathscr{K} \forall x_{1}, x_{2} \in \mathscr{K}, \alpha_{1}, \alpha_{2} \geq 0 . \mathscr{K}$ is called solid if the interior of $\mathscr{K}, \operatorname{int}(\mathscr{K})$, is nonempty, and pointed if $\mathscr{K} \cap$ $(-\mathscr{K})=\{0\}$. A proper cone is a closed, pointed, solid cone. A cone is polyhedral if it can be expressed as the nonnegative combination of a finite number of generating vectors $\omega_{1}, \ldots, \omega_{\mu} \in \mathbb{R}^{n}$ :

$$
\mathscr{K}=\operatorname{cone}(\Omega)=\left\{x=\Omega \alpha=\sum_{i=1}^{\mu} \alpha_{i} \omega_{i}, \alpha_{i} \geq 0\right\},
$$

where $\Omega=\left[\omega_{1} \ldots \omega_{\mu}\right] \in \mathbb{R}^{n \times \mu}, \alpha=\left[\alpha_{1} \ldots \alpha_{\mu}\right]^{T} \in \mathbb{R}_{+}^{\mu}$. It is well-known that alternatively to the "vertices description" (1) for $\mathscr{K}$ one can use the "face description"

$$
\mathscr{K}=\{x \text { s. t. } \Gamma x \geq 0\}, \quad \Gamma \in \mathbb{R}^{\mu \times n} .
$$

The pair $\{\Omega, \Gamma\}$ forms a "double description pair" for $\mathscr{K}$. Let $\mathscr{K}^{*}=\left\{y \in \mathbb{R}^{n}\right.$ s. t. $\left.y^{T} x \geq 0 \forall x \in \mathscr{K}\right\}$ be the dual cone of $\mathscr{K}$. In terms of the double description pair $\{\Omega, \Gamma\}$, we have:

$$
\mathscr{K}^{*}=\left\{y \text { s. t. } y=\Gamma^{T} \beta, \beta \geq 0\right\}=\left\{y \text { s. t. } \Omega^{T} y \geq 0\right\},
$$

i.e., $\left\{\Gamma^{T}, \Omega^{T}\right\}$ is a double description pair for $\mathscr{K}^{*}$.

Given $A \in \mathbb{R}^{n \times n}$, the cone $\mathscr{K}$ is said $A$-invariant if $A \mathscr{K} \subseteq$ $\mathscr{K}$. For an $A$-invariant cone $\mathscr{K}, A$ is said $\mathscr{K}$-positive if $A(\mathscr{K} \backslash\{0\}) \subseteq \operatorname{int}(\mathscr{K})$, i.e., $A$ maps any nonzero element of $\mathscr{K}$ into $\operatorname{int}(\mathscr{K})$. Notice that if $A$ is $\mathscr{K}$-positive then $A$ is $\mathscr{K}$-irreducible, i.e., it does not leave any of the faces of $\mathscr{K}$ invariant (except for $\{0\}$ and $\mathscr{K}$ itself). Theorem 1.3.16 of Berman \& Plemmons (1994) says that $A$ that leaves $\mathscr{K}$ invariant is $\mathscr{K}$-irreducible if and only if $A$ has exactly one (up to scalar multiples) eigenvector in $\mathscr{K}$, and this vector is in $\operatorname{int}(\mathscr{K}) . A$ is $\mathscr{K}$-positive if and only if $A^{T}$ is $\mathscr{K}^{*}$-positive Berman \& Plemmons (1994).

The Perron-Frobenius theorem for invariant polyhedral cones can be found e.g. in Berman \& Plemmons (1994) (Theorem 1.3.26) or Valcher \& Farina (2000) (Theorem 3.3).

Theorem 3 Given $A \in \mathbb{R}^{n \times n}$, the following are equivalent:

(1) $\exists$ a proper $A$-invariant polyhedral cone $\mathscr{K} \in \mathbb{R}^{n}$ for which $A$ is $\mathscr{K}$-positive;

(2) $\rho(A)$ is a simple positive eigenvalue in $\operatorname{sp}(A)$, and for each $\lambda \in \operatorname{sp}(A), \lambda \neq \rho(A),|\lambda|<\rho(A)$.
Furthermore, for the right eigenvector $v$ relative to $\rho(A)$ it holds $v \in \operatorname{int}(\mathscr{K})$.

The following theorem links eventually positive matrices with invariant cones. The notation $A^{\eta} \mathscr{K}$ stands for the $\eta$-th iterated cone of $\mathscr{K}$.

Theorem 4 (Altafini \& Lini (2015), Theorem 5) $A \stackrel{\vee}{>} 0$ if and only if $\exists$ a proper polyhedral A-invariant cone $\mathscr{K}$ for which $A$ is $\mathscr{K}$-positive, and $\mathscr{K}$ is such that $A^{\eta} \mathscr{K} \subset \operatorname{int}\left(\mathbb{R}_{+}^{n}\right) \cup\{0\}$, $\left(A^{T}\right)^{\eta} \mathscr{K}^{*} \subset \operatorname{int}\left(\mathbb{R}_{+}^{n}\right) \cup\{0\} \forall \eta \geq \eta_{o}$.

By construction, the cone $\mathscr{K}$ of Theorem 4 contains no other eigenvector of $A$ than $v$. If instead of $A \stackrel{\vee}{>} 0$ we have the "one-sided" condition $A \in \mathscr{P} \mathscr{F}_{n}$, then Theorem 4 can be replaced by the following corollary.

Corollary 1 (Altafini \& Lini (2015), Corollary 2) $A \in \mathscr{P} \mathscr{F}_{n}$ if and only if $\exists$ a proper polyhedral A-invariant cone $\mathscr{K}$ for which $A$ is $\mathscr{K}$-positive, and $\mathscr{K}$ is such that $A^{\eta} \mathscr{K} \subset$ $\operatorname{int}\left(\mathbb{R}_{+}^{n}\right) \cup\{0\} \forall \eta \geq \eta_{o}$.

\section{Discrete-time eventually positive realizations}

The discrete-time SISO linear system

$$
\begin{aligned}
x(k+1) & =A x(k)+b u(k) \quad k=0,1, \ldots \\
y(k) & =c x(k)
\end{aligned}
$$

is said externally positive if for any nonnegative input sequence $\{u(k)\}_{k=0}^{\infty}$ the forced output (in correspondence of $x(0)=0)$ is nonnegative. A well-known necessary and sufficient condition for external positivity is that the impulse response $\left\{u(k)=\delta_{k}\right\}_{k=0}^{\infty}$ is nonnegative. The system (2) is said (internally, hereafter omitted) positive if for any nonnegative input sequence $\{u(k)\}_{k=0}^{\infty}$ and nonnegative initial condition $x(0)$, the state $x(k)$ and the output $y(k)$ are always nonnegative. A necessary and sufficient condition for positivity of (2) is that $A \geq 0, b \geq 0$ and $c \geq 0$, see Farina \& Rinaldi (2000).

Consider the strictly proper, rational transfer function of order $n$

$$
H(z)=\frac{P(z)}{Q(z)}=\frac{p_{1} z^{n-1}+p_{2} z^{n-2}+\ldots+p_{n-1} z+p_{n}}{z^{n}+q_{1} z^{n-1}+\ldots+q_{n-1} z+q_{n}}
$$

where we assume that $P(z)$ and $Q(z)$ are coprime polynomials. Expanding (3) in terms of the Markov parameters $\{h(i)\}_{i=1}^{\infty}$ we can write

$$
H(z)=\sum_{i=1}^{\infty} h(i) z^{-i}
$$


where the recursive expression for the $\{h(i)\}_{i=1}^{\infty}$ is

$$
h(i)=-\sum_{j=1}^{i-1} q_{i-j} h(j)+p_{i}
$$

A pole of $H(z)$ is called a dominating pole if its modulus is maximum among all poles of $H(z)$. It is called strictly dominating if it is dominating and all other poles of $H(z)$ have strictly smaller modulus.

A triplet $\{A, b, c\}$ is a realization of the strictly proper rational transfer function $H(z)$ if $H(z)=c(z I-A)^{-1} b$. A realization is minimal if and only if $\{A, b\}$ is controllable and $\{A, c\}$ observable. In this case $n$ denotes both the dimension of $A$ and the order of $H(z)$. A realization is positive if $\{A, b, c\}$ is a positive system.

Throughout this paper we will make the following assumption.

Assumption $1 \mathrm{H}(z)$ has a simple, strictly dominating real pole, denoted $\rho$, i.e., $Q(z)$ factorizes as $Q(z)=Q^{\prime}(z)(z-\rho)$.

Under this assumption, any minimal realization $\{A, b, c\}$ has to have $\rho$ as a simple, strictly dominating real eigenvalue, i.e., $\rho=\rho(A)>|\lambda| \forall \lambda \in \operatorname{sp}\{A\}, \lambda \neq \rho(A)$, see Benvenuti $\&$ Farina (2004).

Necessary and sufficient conditions for existence of a positive realization of $H(z)$ are provided in Ohta et al. (1984); Maeda \& Kodama (1981); Farina \& Benvenuti (1995). The formulation in the following Theorem is taken from Anderson et al. (1996) (Theorems 2.1, 2.2 and Remark in between).

Theorem 5 Let $H(z)$ be a rational transfer function with minimal realization $\{A, b, c\}$. Then $H(z)$ has a positive realization if and only if $\exists$ a proper polyhedral cone $\mathscr{K}$ such that
(1) $A \mathscr{K} \subseteq \mathscr{K}$
(2) $b \in \mathscr{K}$;
(3) $c \in \mathscr{K}^{*}$.

In particular, in the case of simple strictly dominant real pole, it is known that a positive realization of some dimension $\mu \geq n$ always exists (Theorem 31 of Farina \& Rinaldi (2000)) hence from Theorem 5, a proper $A$-invariant polyhedral cone $\mathscr{K}$ for which $b \in \mathscr{K}$ and $c \in \mathscr{K}^{*}$ always exists. Once $\mathscr{K}=$ cone $(\Omega)$ has been found, then $\Omega \in \mathbb{R}^{n \times \mu}, \mu \geq n$, can be used to form a $\mu$-dimensional positive realization $\left\{A_{p}, b_{p}, v_{p}\right\}$, where

$$
A \Omega=\Omega A_{p}, b=\Omega b_{p}, c_{p}=c \Omega .
$$

When $\mu>n$, the realization $\left\{A_{p}, b_{p}, v_{p}\right\}$ is nonminimal.
In this paper we introduce a class of minimal realizations which can be used to fill the gap between external positivity and (internal) positivity, at least under Assumption 1. We call these realizations eventually positive.

Definition 4 A realization $\left\{A_{e}, b_{e}, c_{e}\right\}$ is said eventually positive if $A_{e} \stackrel{\vee}{>} 0$ and $\exists$ an $A_{e}$-invariant cone $\mathscr{K}_{e}$ for which $A_{e}$ is $\mathscr{K}_{e}$-positive and such that $b_{e} \in \mathscr{K}_{e}, c_{e} \in \mathscr{K}_{e}^{*}$.

The eventually positive realizations we are interested in are minimal, i.e., $A_{e} \in R^{n \times n}, b_{e}, c_{e}^{T} \in \mathbb{R}^{n}$. Notice that from Theorem $1, A_{e} \stackrel{\vee}{>} 0$ implies $\rho\left(A_{e}\right)>0$ is a simple strictly dominating real eigenvalue, i.e., Assumption 1 holds. From Theorem 3 , in this case, a proper polyhedral cone $\mathscr{K}_{e}$ for which $A_{e}$ is $\mathscr{K}_{e}$-positive always exists.

\subsection{A constructive procedure}

From Definition 4 and Theorem 1, a necessary condition for a minimal realization $\{A, b, c\}$ of $H(z)$ to be eventually positive is that $v>0$ and $w>0$, where $v$ and $w$ are the right and left eigenvectors of $A$ relative to $\rho$.

Recall that if $M$ is an invertible matrix, the change of basis performed via $M$ yields:

$$
\begin{aligned}
A^{\prime} & =M^{-1} A M \\
b^{\prime} & =M^{-1} b \\
c^{\prime} & =c M \\
v^{\prime} & =M^{-1} v \\
w^{\prime} & =M^{T} w .
\end{aligned}
$$

In this section we give a procedure to construct a minimal eventually positive realization of $H(z)$ satisfying Assumption 1. Let us consider the realization $\left\{A_{o}, b_{o}, c_{o}\right\}$

$$
\begin{gathered}
A_{o}=\left[\begin{array}{ccccc}
0 & 1 & 0 & \ldots & 0 \\
0 & 0 & 1 & & \\
\vdots & & & \ddots & \\
0 & & & 0 & 1 \\
-q_{n} & -q_{n-1} & \ldots & -q_{2} & -q_{1}
\end{array}\right], \\
b_{o}=\left[\begin{array}{c}
h(1) \\
h(2) \\
\vdots \\
h(n-1) \\
h(n)
\end{array}\right], \quad c_{o}=\left[\begin{array}{llll}
1 & 0 & \ldots & 0
\end{array}\right] .
\end{gathered}
$$


which is sometimes called Markov observability canonical form because of the Markov parameters appearing in $b_{o}$. In this case the polyhedral cone (hereafter $\mathscr{K}_{o}$ ) is called the Markov cone (Anderson et al. , 1996; Farina \& Rinaldi, 2000) and it is generated by

$$
\Omega_{o}=\left[\begin{array}{cccc}
h(1) & h(2) & \ldots & h(\mu) \\
\vdots & \vdots & & \vdots \\
h(n) & h(n+1) & \ldots & h(\mu+n-1)
\end{array}\right]
$$

For the Markov observability canonical form, $v_{o}>0$, while $w_{o}$ has no fixed sign pattern. The condition $v_{o}>0$ guarantees that $A_{o} \in \mathscr{P} \mathscr{F}_{n}$. From Corollary 1, it also guarantees that $A^{\eta} \mathscr{K}_{o}$ belongs to $\operatorname{int}\left(\mathbb{R}_{+}^{n}\right)$ for sufficiently high $\eta \in \mathbb{N}$. It however does not imply that also $A_{o}^{T} \in \mathscr{P} \mathscr{F}_{n}$, nor that $\left(A^{T}\right)^{\eta} \mathscr{K}_{o}^{*} \subset \operatorname{int}\left(\mathbb{R}_{+}^{n}\right)$ for any $\eta$. For that, one may have to "tilt" the $\left\{A_{o}, b_{o}, c_{o}\right\}$ realization, rendering $w_{o}$ positive while not changing the sign of $v_{o}$. This can be done through operations with elementary matrices as in the following algorithm.

\section{Algorithm 1.}

Input: $A=A_{o}, b=b_{o}, c=c_{o}, v=v_{o}, w=w_{o}$.

Step 0: To begin with, notice that possibly by multiplying by $-I_{n}$, we can always assume that $w$ has at least $n / 2$ (or $(n+1) / 2$ if $n$ is odd) nonnegative entries.

Step 1: Consider an index $i$ for which $w_{i} \leq 0$. Two cases are possible:

Case 1: $\exists$ index $j \neq i, j \neq 1$, for which $w_{j}>0$ and

$$
0 \leq-\frac{w_{i}}{w_{j}}<\frac{v_{j}}{v_{i}}
$$

where by construction $-\frac{w_{i}}{w_{j}} \geq 0$ and $\frac{v_{j}}{v_{i}}>0$. In this case, choosing

$$
M^{(\xi)}=\left[\begin{array}{ccccc}
1 & 0 & \ldots & & 0 \\
0 & 1 & & & \\
\vdots & \ddots & m_{j i}^{(\xi)} & \\
& & \ddots & 0 \\
0 & & & 0 & 1
\end{array}\right]=I_{n}+\left[m_{j i}^{(\xi)}\right]
$$

where

$$
m_{j i}^{(\xi)} \in\left(-\frac{w_{i}}{w_{j}}, \frac{v_{j}}{v_{i}}\right)>0,
$$

one gets in (6):

$$
\begin{aligned}
w_{i}^{\prime} & =w_{i}+m_{j i}^{(\xi)} w_{j}>0 \\
w_{j}^{\prime} & =w_{j}>0 \\
v_{i}^{\prime} & =v_{i}>0 \\
v_{j}^{\prime} & =-m_{j i}^{(\xi)} v_{i}+v_{j}>0
\end{aligned}
$$

i.e., the sign of $w_{i}$ has become positive.

Case 2: No index $j \neq i$ exists for which $w_{j}>0$ and (7) holds. In this case, choosing any index $j \neq i, j \neq 1$ for which $w_{j}>0$, it is possible to increase the corresponding $v_{j}$ by combining the $j$-th row with any of the rows (indexed by $k$ ) having $w_{k}>0$ and $c_{k}=0$ (at least $n / 2-1$ such rows exist by construction). In this case the elementary matrix that can be used is

$$
M^{(\xi)}=I_{n}+\left[m_{j k}^{(\xi)}\right]
$$

with $-\frac{w_{k}}{w_{j}}<m_{j k}^{(\xi)}<0$, which yields

$$
\begin{aligned}
w_{j}^{\prime} & =w_{j}>0 \\
0<w_{k}^{\prime} & =m_{j k}^{(\xi)} w_{j}+w_{k}<w_{k} \\
v_{j}^{\prime} & =v_{j}-m_{j k}^{(\xi)} v_{k}>v_{j}>0 \\
v_{k}^{\prime} & =v_{k}>0 .
\end{aligned}
$$

If $v_{j}^{\prime}$ is now sufficiently big that (7) holds, then Case 1) applies. If not, then another index $k$ must be chosen and Case 2) iterated.

Step 2: Repeat until $w_{j}^{\prime}>0 \forall j=1, \ldots, n$, or until Step 1 becomes unfeasible.

Step 3: If $w^{\prime}>0$ the Algorithm terminated successfully. In this case, if $M^{\left(\xi_{1}\right)}, \ldots, M^{\left(\xi_{\psi}\right)}$ are the $\xi_{\psi}$ elementary matrices used in Step 1 above, then the change of basis $M=$ $M^{\left(\xi_{1}\right)} \cdots M^{\left(\xi_{\psi}\right)}$ yields the sought realization $\left\{A_{e}, b_{e}, c_{e}\right\}$.

The following Proposition shows that indeed $\left\{A_{e}, b_{e}, c_{e}\right\}$ constructed in this way is a minimal eventually positive realization of $H(z)$.

Proposition 1 Consider a strictly proper rational transfer function $\mathrm{H}(z)$ obeying Assumption 1. Assume Algorithm 1 terminates successfully and consider the corresponding realization $\left\{A_{e}, b_{e}, c_{e}\right\}$. Then $\exists$ a proper polyhedral $A_{e}$-invariant cone $\mathscr{K}_{e}$ such that:

(1) $A^{\eta} \mathscr{K}_{e} \subset \operatorname{int}\left(\mathbb{R}_{+}^{n}\right) \cup\{0\}$ for $\eta \geq \eta_{o}$;

(2) $\left(A^{T}\right)^{\eta} \mathscr{K}_{e}^{*} \subset \operatorname{int}\left(\mathbb{R}_{+}^{n}\right) \cup\{0\}$ for $\eta \geq \eta_{o}$;

(3) $A_{e}$ is $\mathscr{K}_{e}$-positive;

(4) $b_{e} \in \mathscr{K}_{e}$;

(5) $c_{e} \in \mathscr{K}_{e}^{*}$.

Consequently, $\left\{A_{e}, b_{e}, c_{e}\right\}$ is a minimal eventually positive realization of $H(z)$. 
Proof. Since $A_{e}>0$, the first 3 items follow from Theorem 4 . Consider the minimal realization in Markov observability canonical form $\left\{A_{o}, b_{o} c_{o}\right\}$. By construction $b_{o} \in \mathscr{K}_{o}$ and $c_{o} \in \mathscr{K}_{o}^{*}$. If Algorithm 1 terminates successfully then there exists a change of basis $M$ such that $A_{e}=M^{-1} A_{o} M, b_{e}=$ $M^{-1} b_{o}, c_{e}=c_{o} M$. Applying $M$ to the double description pair $\left(\Omega_{o}, \Gamma_{o}\right)$ of the Markov cone: $\Omega_{e}=M^{-1} \Omega_{o}$ and $\Gamma_{e}=M^{T} \Gamma_{o}$. Since $b_{o}=\Omega \alpha$ for some $\alpha \in \mathbb{R}_{+}^{\mu}$, it is $b_{e}=M^{-1} \Omega_{o} \alpha=\Omega_{e} \alpha$, i.e., $b_{e} \in \mathscr{K}_{e}$. Similarly, from $c^{T}=\Gamma_{o} \beta$ for some $\beta \in \mathbb{R}_{+}^{\mu}$, it is $c_{e}^{T}=M^{T} \Gamma_{o} \beta=\Gamma_{e} \beta$ i.e., $c_{e} \in \mathscr{K}_{e}^{*}$. Therefore $\left\{A_{e}, b_{e}, c_{e}\right\}$ is an eventually positive realization of $H(z)$. Minimality of this realization follows from invariance of the minimality property to a change of basis.

It follows from Proposition 1 and from $A_{e}$-invariance of $\mathscr{K}_{e}$ that for the reachability cone $\mathscr{R}_{e}=\operatorname{cone}\left(b_{e}, A_{e} b_{e}, A_{e}^{2} b_{e}, \ldots\right)$ of the system $\left\{A_{e}, b_{e}, c_{e}\right\}$ it holds:

$$
\mathscr{R}_{e} \subset \mathscr{K}_{e}
$$

Recall that the solution of a linear discrete-time system can be split into free and forced evolution. For $\left\{A_{e}, b_{e}, c_{e}\right\}$ one gets:

$$
x(k)=x_{o}(k)+x_{f}(k)=A_{e}^{k} x(0)+\sum_{j=0}^{k-1} A_{e}^{k-j-1} b_{e} u(j) .
$$

Proposition 2 Consider a minimal eventually positive realization $\left\{A_{e}, b_{e}, c_{e}\right\}$ of $H(z)$. Then $\exists \eta_{o} \in \mathbb{N}$ such that $x_{o}(k) \geq$ $0 \forall k \geq \eta_{o}, \forall x(0) \in \mathbb{R}_{+}^{n}$. If in addition $\mathscr{R}_{e} \subset \mathbb{R}_{+}^{n}$, then $x(k) \geq$ $0 \forall k \geq \eta_{o}, \forall x(0) \in \mathbb{R}_{+}^{n}, \forall u(k) \in \mathbb{R}_{+}$.

Proof. $A_{e} \stackrel{\vee}{>} 0$ implies $A_{e}^{k}>0 \forall k \geq \eta_{o}$, hence $x_{o}(k)=$ $A_{e}^{k} x(0) \geq 0 \forall x(0) \in \mathbb{R}_{+}^{n}$. Since $b_{e}$ can have any sign, an analogous property does not hold in general for the forced evolution $x_{f}(k)$. However, $\mathscr{R}_{e} \subset \mathbb{R}_{+}^{n}$ implies $x_{f}(k) \geq 0 \forall k$.

The following theorem summarizes the main result so far.

Theorem 6 Consider a strictly proper rational transfer function $H(z)$ obeying Assumption 1. If $H(z)$ admits a minimal eventually positive realization then it is externally positive. Conversely, if $H(z)$ is externally positive and Algorithm 1 terminates successfully, then $H(z)$ has a minimal eventually positive realization.

Proof. Consider an eventually positive realization and the corresponding $A_{e}$-invariant polyhedral cone $\mathscr{K}_{e}$. From $b_{e} \in$ $\mathscr{K}_{e}$ and $c_{e} \in \mathscr{K}_{e}^{*}$, it follows that $c_{e} b_{e} \geq 0 . A_{e}$-invariance then implies that $A_{e}^{k} b_{e} \in \mathscr{K}_{e} \forall k=1,2, \ldots$ and therefore that $c_{e} A_{e}^{k} b_{e} \geq 0$. Conversely, Proposition 1 implies that success in Algorithm 1 yields an eventually positive realization $\left\{A_{e}, b_{e}, c_{e}\right\}$. Minimality also follows from Proposition 1 .
According to Definition 4 , in an eventually positive realization no sign constraint is imposed on $b_{e}$ and $c_{e}$. It is however easy to modify Algorithm 1 adding as constraints $b_{e} \geq 0$ and $c_{e} \geq 0$.

\section{Algorithm 2.}

Input: $A=A_{o}, b=b_{o}, c=c_{o}, v=v_{o}, w=w_{o}$.

Step 0: same as in Algorithm 1.

Step 1: Case 1: similar to Algorithm 1, but replacing (7) with

$$
0 \leq-\frac{w_{i}}{w_{j}}<\min \left(\frac{b_{j}}{b_{i}}, \frac{v_{j}}{v_{i}}\right)
$$

where by construction $\frac{b_{j}}{b_{i}}>0$ when $b_{i} \neq 0\left(\frac{b_{j}}{b_{i}}=+\infty\right.$ otherwise, also admissible). In this case, $M^{(\xi)}=I_{n}+$ $\left[m_{j i}^{(\xi)}\right]$ can be chosen as

$$
m_{j i}^{(\xi)} \in\left(-\frac{w_{i}}{w_{j}}, \min \left(\frac{b_{j}}{b_{i}}, \frac{v_{j}}{v_{i}}\right)\right)>0,
$$

Case 2: same as Algorithm 1.

Step 2 \& 3: same as Algorithm 1.

Proposition 3 Consider a strictly proper rational transfer function $H(z)$ obeying Assumption 1. Assume Algorithm 2 terminates successfully and consider the corresponding realization $\left\{A_{e}, b_{e}, c_{e}\right\}$. Then Proposition 1 holds, and in addition:

(1) $b_{e} \geq 0$;

(2) $c_{e} \geq 0$.

Proof. If Algorithm 2 is successful then so is Algorithm 1, hence Proposition 1 holds. Since $b_{o}$ contains Markov coefficients, $b_{o} \geq 0$ and $c_{o} \geq 0$. By construction, the condition (12) guarantees that in Case 1 , when applying $M^{(\xi)}=I_{n}+\left[m_{j i}^{(\xi)}\right]$, alongside (9) one gets

$$
\begin{aligned}
b_{i}^{\prime} & =b_{i}>0 \\
b_{j}^{\prime} & =-m_{j i}^{(\xi)} b_{i}+b_{j}>0 \\
c_{i}^{\prime} & =c_{i} \\
c_{j}^{\prime} & =c_{j}=0
\end{aligned}
$$

i.e., the sign of $w_{i}$ has become positive while the sign of $b$ and $c$ is preserved at each iteration. Similarly, in Case 2, since $t_{j k}^{(\xi)}<0$, in addition to $(10), M^{(\xi)}=I_{n}+\left[m_{j k}^{(\xi)}\right]$ yields

$$
\begin{aligned}
b_{j}^{\prime} & =b_{j}-m_{j k}^{(\xi)} b_{k}>b_{j}>0 \\
b_{k}^{\prime} & =b_{k}>0 \\
c_{j}^{\prime} & =c_{j}=0 \\
c_{k}^{\prime} & =c_{k}=0
\end{aligned}
$$


i.e., the sign of $b$ and $c$ is preserved.

Remark 1 Notice that $b_{e} \geq 0 \not \mathscr{R}_{e} \subset \mathbb{R}_{+}^{n}$, and hence $\not$ $x(k) \geq 0$ for $k \geq \eta_{o}$. It follows however from Proposition 2 that the impulse response of a minimal eventually positive realization with $b_{e} \geq 0$ and $c_{e} \geq 0$ is such that $x(t) \geq 0$ $\forall k \geq \eta_{o}, \forall x(0) \in \mathbb{R}_{+}^{n}$. In other words, while the impulse response completely characterizes external positivity it is not enough to characterize internal positivity, as expected.

In Guidorzi (2014) the author considers a class of minimal realizations of externally positive systems which he calls "quasi-positive", meaning realizations $\left\{A_{q}, b_{q}, c_{q}\right\}$ such that $b_{q} \geq 0, c_{q} \geq 0$ and $\mathscr{R}_{q} \subseteq \mathbb{R}_{+}^{n}$. It follows that for such realization $x_{f}(k) \geq 0 \forall k \geq 0$, while the sign of $x_{o}(k)$ cannot be guaranteed, not even asymptotically (unless, trivially, also $x(0) \in \mathscr{R}_{q}$ ), i.e., a "quasi-positive" realization may have state variables which never become positive even if $x(0) \geq 0$. As an example, consider the Markov observability canonical form $\left\{A_{o}, b_{o}, c_{o}\right\}$ which by construction is always one such realization for any externally positive system. For it $v_{o}>0$, hence $A_{o} \in \mathscr{P} \mathscr{F}_{n}$ when Assumption 1 holds. Therefore Corollary 1 holds but not necessarily Theorem 4 , as the sign of $w_{o}$ is not fixed a priori. As a consequence, asymptotically, the free evolution

$$
\lim _{k \rightarrow \infty} x_{o}(k)=\frac{v_{o} w_{o}^{T} x(0)}{w_{o}^{T} v_{o}}
$$

can assume any sign even if $x(0) \geq 0$.

For eventually positive realizations, instead, the following stronger characterization holds.

Proposition 4 Consider a minimal eventually positive realization $\left\{A_{e}, b_{e}, c_{e}\right\}$ of $H(z)$ such that $b_{e} \geq 0$ and $c_{e} \geq 0$. Then $\exists \eta_{o} \in \mathbb{N}$ such that $x(k) \geq 0 \forall k \geq \eta_{o}, \forall x(0) \in \mathbb{R}_{+}^{n}$. If in addition $\mathscr{R}_{e} \subset \mathbb{R}_{+}^{n}$, then $x_{f}(\bar{k}) \geq 0 \forall k \geq 0, \forall u(k) \in \mathbb{R}_{+}$.

Proof. The proof is analogous to that of Proposition 2, with the extra condition $b_{e} \geq 0$ guaranteeing now that $x_{f}(k) \geq 0$ $\forall k \geq \eta_{o}$. When in addition $\mathscr{R}_{e} \subset \mathbb{R}_{+}^{n}$, then obviously $x_{f}(k) \geq$ $0 \forall k \geq 0, \forall u(k) \in \mathbb{R}_{+}$.

\subsection{Examples}

Example 1 Consider the following transfer function

$H(z)=\frac{0.1048 z^{3}+0.1312 z^{2}-0.02171 z-0.01499}{z^{5}-0.9631 z^{4}-0.05818 z^{3}+0.03503 z^{2}-0.01051 z-0.003276}$

As $\rho=1$, the Markov parameters are all nonnegative and "stabilize" at $\lim _{k \rightarrow \infty} h(k)=0.193$, we can conclude that
$H(z)$ is externally positive. The Markov observability canonical form $\left\{A_{o}, b_{o}, c_{o}\right\}$ has $v_{o}=\mathbf{1}$ and

$$
w_{o}=\left[\begin{array}{c}
0.0033 \\
0.0138 \\
-0.0212 \\
0.0369 \\
0.9990
\end{array}\right]
$$

hence the dual cone $\left(A^{T}\right)^{\eta} \mathscr{K}_{e}^{*}$ is not contained in $\mathbb{R}_{+}^{5}$ for any $\eta$. However, transforming this canonical form as in (6) with $M_{1}=I_{5}+\left[m_{43}\right], m_{43}=0.6076$, one gets

$$
A_{e_{1}}=\left[\begin{array}{ccccc}
0 & 1 & 0 & 0 & 0 \\
0 & 0 & 1 & 0 & 0 \\
0 & 0 & 0.6076 & 1 & 0 \\
0 & 0 & -0.3692 & -0.6076 & 1 \\
0.0033 & 0.0105 & 0.0003 & 0.0582 & 0.9631
\end{array}\right]
$$

which has positive right and left dominant eigenvectors

$$
v_{e_{1}}=\left[\begin{array}{c}
1 \\
1 \\
1 \\
0.3924 \\
1
\end{array}\right], \quad w_{e_{1}}=\left[\begin{array}{c}
0.0033 \\
0.0138 \\
0.0012 \\
0.0369 \\
1
\end{array}\right]
$$

$A_{e_{1}}$ is eventually positive, with a power index $\eta_{o}=8$, hence the $\left\{A_{e_{1}}, b_{e_{1}}, c_{e_{1}}\right\}$ realization is eventually positive. For it

$$
b_{e_{1}}=\left[\begin{array}{c}
0 \\
0.1048 \\
0.2321 \\
0.0669 \\
0.1951
\end{array}\right] \quad \text { and } \quad c_{e_{1}}=c_{o}
$$

while the input matrix in the Markov observability canonical form was:

$$
b_{o}=\left[\begin{array}{c}
0 \\
0.1048 \\
0.2321 \\
0.2079 \\
0.1951
\end{array}\right] .
$$

In this case, altering (lowering) one of the Markov parameters has been enough to "compensate" the negative entry 
in the left eigenvector of $A_{o}$. A simulation of the impulse response for this eventually positive realization is shown in Fig. 1. It shows the transient violation of positivity of the state vector, which is not "visible" at the output. Since $b_{e_{1}} \geq 0$ and $A_{e_{1}}^{k} b_{e_{1}} \geq 0 \forall k$, in this case strict dominance implies that the violation of positivity must necessarily be transient for any nonnegative $\{u(k)\}_{k=0}^{\infty}$.

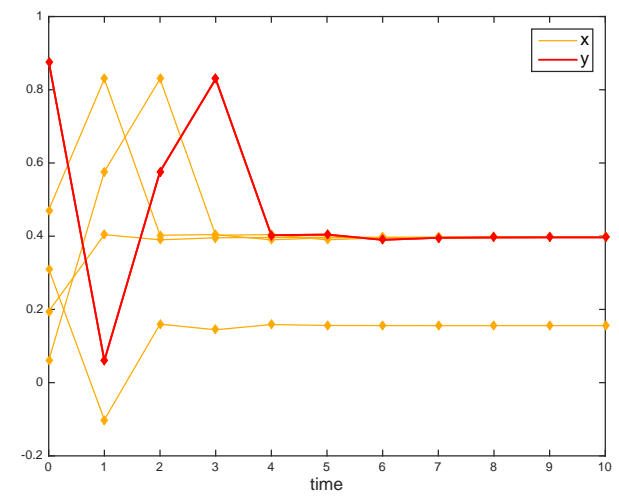

Fig. 1. Example 1. When the initial conditions are nonzero but positive, the impulse response yields a positive output, but one of the state variables has a transient which does not remain positive.

Example 2 It is worth observing that the power index of an eventually positive realization may vary among different eventually positive realizations of the same externally positive transfer function. Consider again Example 1. If instead of using the change of basis $M_{1}$ we use the following $M_{2}=I_{5}+\left[m_{53}\right]$ with $m_{53}=0.1032$, then we get another eventually positive realization, with

$$
A_{e_{2}}=\left[\begin{array}{ccccc}
0 & 1 & 0 & 0 & 0 \\
0 & 0 & 1 & 0 & 0 \\
0 & 0 & 0 & 1 & 0 \\
0 & 0 & 0.1032 & 0 & 1 \\
0.0033 & 0.0105 & 0.0643 & -0.0450 & 0.9631
\end{array}\right]
$$

$$
b_{e_{2}}=\left[\begin{array}{c}
0 \\
0.1048 \\
0.2321 \\
0.2079 \\
0.1711
\end{array}\right], \quad c_{e_{2}}=c_{o}
$$

of right and left dominant eigenvectors

$$
v_{e_{2}}=\left[\begin{array}{c}
1 \\
1 \\
1 \\
1 \\
0.8968
\end{array}\right], \quad w_{e_{2}}=\left[\begin{array}{c}
0.0033 \\
0.0138 \\
0.0819 \\
0.0369 \\
1
\end{array}\right]
$$

Also this time "tilting" one of the coordinates is enough to achieve positivity of the left dominant eigenvector. However by acting on the fifth state component (instead of the fourth of Example 1) we obtain an eventually positive realization whose power index is $5\left(A_{e_{2}}^{k} \geq 0\right.$ already at $k=2$, then we must take further nowers to get all nositive entries).

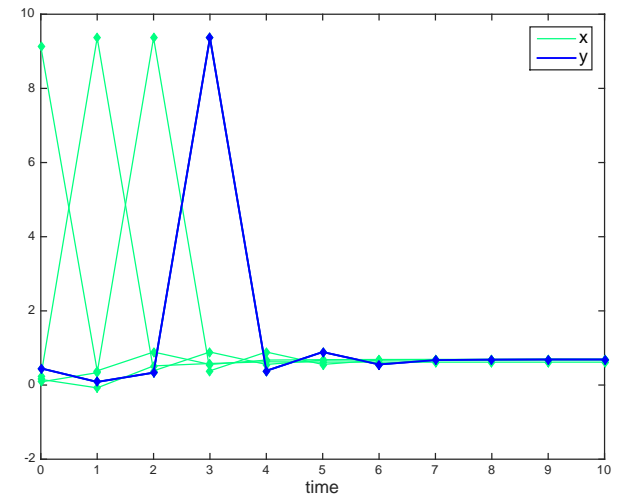

Fig. 2. Example 2. For the same $H(s)$ of Example 1, changing the transformation matrix $M$ may lead to a different eventually positive realization.

\section{Recovering positivity through downsampling}

The extra conditions $b_{e} \geq 0$ and $c_{e} \geq 0$ imposed in Algorithm 2 can be used to obtain minimal positive realizations for decimated subsequences of the Markov parameters.

Theorem 7 Consider a strictly proper rational transfer function $H(z)$ satisfying Assumption 1 and the corresponding sequence of Markov parameters $\{h(k)\}_{k=1}^{\infty}$. Assume $\exists r \in \mathbb{N}, r \neq 0$, such that $r \operatorname{Im}\left[\lambda_{i}-\lambda_{j}\right] \neq 2 \pi \xi$, $\xi= \pm 1, \pm 2, \ldots$, for all distinct poles $\lambda_{i}$ and $\lambda_{j}$ of $H(z)$ having $\operatorname{Re}\left[\lambda_{i}-\lambda_{j}\right]=0$. If $H(z)$ admits a minimal eventually positive realization $\left\{A_{e}, b_{e}, c_{e}\right\}$ such that $b_{e} \geq 0$ and $c_{e} \geq 0$, then $\exists \sigma \in \mathbb{N}$ such that the decimated sequence of Markov parameters $\left\{h_{s}(k)=h((k-1) s+1)\right\}_{k=1}^{\infty}$ admits a minimal positive realization $\left\{A_{s}, b_{e}, c_{e}\right\}$ with $A_{s}=A_{e}^{\sigma}$.

Proof. Consider a minimal eventually positive realization $\left\{A_{e}, b_{e}, c_{e}\right\}$ of $H(z)$. If $\eta_{o}$ is the power index of $A_{e}, A_{e}^{\sigma}>0$ $\forall \sigma \in \mathbb{N}, \sigma \geq \eta_{o}$. Then each decimated sequence $\left\{h_{s}(k)\right\}_{k=1}^{\infty}$ admits a positive realization $\left\{A_{s}, b_{e}, c_{e}\right\}$, where $A_{s}=A_{e}^{\sigma}$. In fact, from $h(k)=c_{e} A_{e}^{k-1} b_{e}$, one gets $h_{s}(k)=h((k-1) \sigma+$ 1) $=c_{e} A_{e}^{(k-1) \sigma} b_{e}=c_{e} A_{s}^{k-1} b_{e}$. In order for $\left\{A_{s}, b_{e}, c_{e}\right\}$ to be minimal, it must be $\left\{A_{s}, b_{e}\right\}$ controllable, and $\left\{A_{s}, c_{e}\right\}$ observable. Since the system is SISO and $\left\{A_{e}, b_{e}\right\}$ controllable, $\left\{A_{e}, c_{e}\right\}$ observable by construction, the geometric multiplicity of each distinct eigenvalue has to be 1 (i.e., there is a single Jordan block associated to each distinct eigenvalue). Denote $\lambda_{1}, \ldots, \lambda_{m}, m \leq n$, the distinct eigenvalues of $A_{e}$, of multiplicities $\zeta_{1}, \ldots, \zeta_{m}$. Since the eigenvalues of $A_{s}$ are $\lambda_{i}^{\sigma}$, $i=1, \ldots, m$, whenever a merge happens, i.e., $\lambda_{i}^{\sigma}=\lambda_{j}^{\sigma}$ for $i, j \in\{1, \ldots, m\}, i \neq j$, then controllability and observability of $\left\{A_{s}, b_{e}, c_{e}\right\}$ are lost, i.e., $\left\{A_{s}, b_{e}, c_{e}\right\}$ is a nonminimal 
realization of $\left\{h_{s}(k)\right\}_{k=1}^{\infty}$ (the Markov subsequence "looses rank" because of the decimation). However, if $\exists$ nonzero $r \in \mathbb{N}$ such that $r \operatorname{Im}\left[\lambda_{i}-\lambda_{j}\right] \neq 2 \pi \xi \xi= \pm 1, \pm 2, \ldots$, for all distinct eigenvalues of $A_{e}$ such that $\operatorname{Re}\left[\lambda_{i}-\lambda_{j}\right]=0$, then $h(k)$ and $h_{s}(k)$ admit minimal realizations of the same dimension. In this case, an admissible decimation factor is $\sigma=\min _{k \in \mathbb{N}}$ s.t. $r k \geq \eta_{o}$ and $r k \operatorname{Im}\left[\lambda_{i}-\lambda_{j}\right] \neq 2 \pi \xi, \xi= \pm 1, \pm 2, \ldots$.

Example 3 Consider again Example 1 and the $\left\{A_{e_{1}}, b_{e_{1}}, c_{e_{1}}\right\}$ eventually positive realization. Since the power index is $8, A_{s_{1}}=A_{e_{1}}^{8}$ and $\left\{A_{s_{1}}, b_{e_{1}}, c_{e_{1}}\right\}$ is a minimal positive realization of the $\left\{h_{8}(k)=h((8 k-7)\}_{k=1}^{\infty}\right.$ subsequence of Markov parameters. By plotting the resulting dynamics in correspondence of the same nonzero initial condition plus impulse input of Fig. 1, we clearly see (Fig. 3) that the downsampling "neglects" the transient response and hence avoids one or more of its states to become temporarily negative. Analogous considerations hold for the realization $\left\{A_{e_{2}}, b_{e_{2}}, c_{e_{2}}\right\}$ (the decimation factor used in passing from Fig. 2 to Fig. 3 is $\sigma=2$ ).
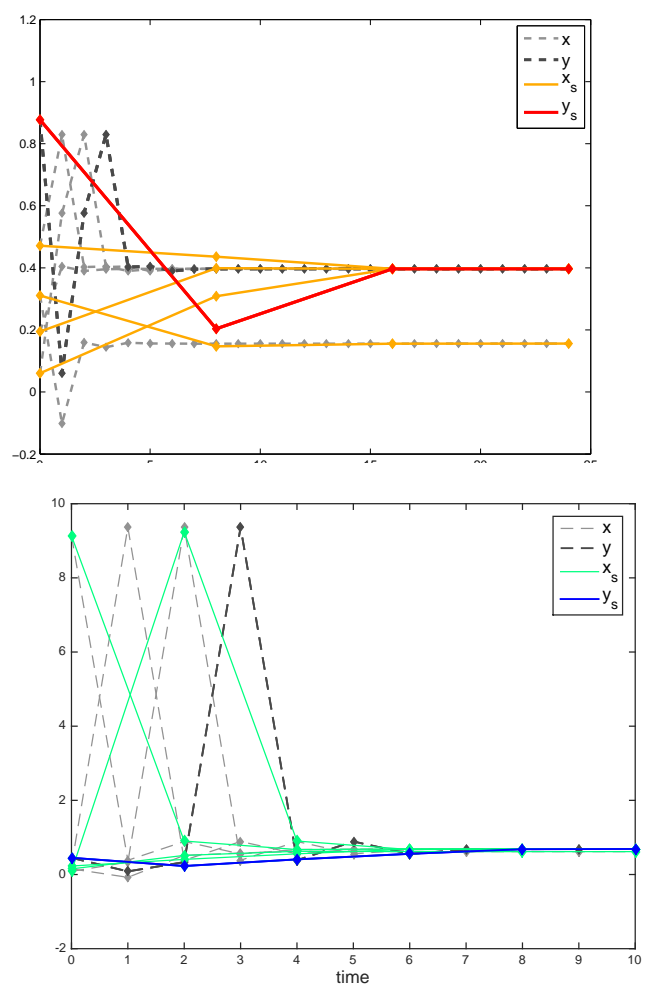

Fig. 3. Example 3. Dynamics of a downsampled minimal eventually positive realization. The response of the eventually positive system of Examples 1 (top) and 2 (bottom) and of the corresponding downsampled positive counterpart from the same initial condition are shown. The state and output of the downsampled positive realization $\left\{A_{s_{i}}, b_{e_{i}}, c_{e_{i}}\right\}$ are denoted resp. $x_{s}$ and $y_{s}$. The impulse response yields a positive output in both systems, and the downsampled system is also positive, but its transient contains less information than the original system.

In Theorem 7 the Markov parameters $h_{s}(\cdot)$ are a decimated sequence of the original Markov parameters $h(\cdot)$. This automatically means that the downsampled impulse is represented as $u(k)=\delta(k)$. It is in principle possible to downsample using other input interpolation techniques such as for example using a Zero-Order Hold $(\mathrm{ZOH})$ methodology. The following remark and corollary show however that such an approach requires extra assumptions to be well-posed.

Remark 2 If $\left\{A_{e}, b_{e}, c_{e}\right\}$ is a minimal eventually positive realization of $H(z)$, then a $\mathrm{ZOH}$ downsampling of (11) by a factor $\sigma \in \mathbb{N}$ yields

$$
\begin{aligned}
A_{\mathrm{ZOH}, s} & =A_{e}^{\sigma} \\
b_{\mathrm{ZOH}, s} & =\sum_{j=0}^{\sigma-1} A_{e}^{\sigma-j-1} b_{e} \\
c_{\mathrm{ZOH}, s} & =c_{e} .
\end{aligned}
$$

The realization $\left\{A_{\mathrm{ZOH}, s}, b_{\mathrm{ZOH}, s}, c_{\mathrm{ZOH}, s}\right\}$ need not be positive. For it however, the Markov parameters are

$$
h_{\mathrm{ZOH}, s}(k)=c_{\mathrm{ZOH}, s} A_{\mathrm{ZOH}, s}^{k-1} b_{\mathrm{ZOH}, s}=c_{e} A_{e}^{(k-1) \sigma} \sum_{j=0}^{\sigma-1} A_{e}^{\sigma-j-1} b_{e}
$$

i.e., they are not a subsequence of the original $c_{e} A_{e}^{k-1} b_{e}$.

When $b_{\mathrm{ZOH}, s} \geq 0$ we have the following straightforward corollary of Theorem 7 .

Corollary 2 Consider a minimal eventually positive realization $\left\{A_{e}, b_{e}, c_{e}\right\}$ of $H(z)$ such that $b_{e} \geq 0$ and $c_{e} \geq 0$. Assume $\exists r \in \mathbb{N}, r \neq 0$, such that $r \operatorname{Im}\left[\lambda_{i}-\lambda_{j}\right] \neq 2 \pi \xi, \xi=$ $\pm 1, \pm 2, \ldots$, for all distinct eigenvalues $\lambda_{i}$ and $\lambda_{j}$ of $A_{e}$ having $\operatorname{Re}\left[\lambda_{i}-\lambda_{j}\right]=0$. If $\mathscr{R}_{e} \subset \mathbb{R}_{+}^{n}$, then for $\sigma \geq \eta_{o}$ the $Z O H$ downsampled realization (13) is positive and minimal.

\section{Continuous time eventually positive realizations}

In a continuous-time linear system, external positivity of $H(s)$ corresponds to the impulse response function $h(t)$ being nonnegative: $h(t) \geq 0 \forall t \geq 0$. For what concerns internal positivity, the matrix $A$ has to be modified to satisfy the Metzler condition. A necessary and sufficient condition for a realization $\{A, b, c\}$ to be positive is in fact that $A$ Metzler, $b \geq 0$ and $c \geq 0$, see Farina \& Rinaldi (2000). For a transfer function $\bar{H}(s)$, existence of a positive realization is given by the analogue of Theorem 5 , which can be easily obtained from Theorem 4 of Ohta et al. (1984).

Theorem 8 Let $H(s)$ be a strictly proper rational transfer function with minimal realization $\{A, b, c\}$. Then $H(s)$ has a positive realization if and only if

(1) $(A+d I) \mathscr{K} \subseteq \mathscr{K}$ for some $d \geq 0$;

(2) $b \in \mathscr{K}$; 
(3) $c \in \mathscr{K}^{*}$.

In this case, if $\mathscr{K}=\operatorname{cone}(\Omega)$, from $(A+d I) \Omega=\Omega A_{+}$, with $A_{+} \geq 0$, one gets that the positive realization is $\left\{A_{p}=A_{+}-\right.$ $\left.d I, \bar{b}_{p}, c_{p}\right\}$, where

$$
A \Omega=\Omega A_{p}, \quad b=\Omega b_{p}, \quad c_{p}=c \Omega .
$$

Starting from a realization $\{A, b, c\}$ of $H(s)$, denote $H_{d}(s-$ $d)=c(s I-A-d I)^{-1} b=c((s-d) I-A)^{-1} b$ for some $d \geq 0$. If $h(t)=c e^{A t} b \geq 0$, then also $c e^{(A+d I) t} b=c e^{A t} e^{d t} b \geq 0$ for any $t \geq 0$, i.e., the extra factor $d I$ does not alter the external positivity of the transfer function. Another necessary and sufficient condition for existence of a positive realization in continuous-time is then the following.

Theorem 9 (Anderson et al. (1996), Theorem 5.1) A strictly proper rational transfer function $H(s)$ has a positive realization if and only if

(1) $\exists d \geq 0$ such that $H_{d}(s-d)$ has nonnegative Markov parameters, i.e., if $H_{d}(s-d)=\sum_{j=1}^{\infty} h_{d}(j)(s-d)^{-j}$, then $h_{d}(j) \geq 0 \forall j=1,2, \ldots$;

(2) there is a unique (possibly multiple) pole of $H(s)$ with maximal real part and the pole is real.

The class of $H(s)$ can be narrowed down in a manner similar to the discrete-time case by restricting to $H(s)$ with a simple dominant pole.

Assumption $2 H(a)$ has a simple real pole with real part strictly bigger than all other poles of $H(s)$.

From Theorem 9, under Assumption 2, existence of a positive realization $\{A, b, c\}$ amounts to nonnegativity of the Markov parameters $h_{d}(k)=c(A-d I)^{k-1} b$ for a suitable $d \geq 0$.

Also eventual positivity is affected by presence of the Metzler condition.

Definition 5 A (continuous-time) realization $\left\{A_{e}, b_{e}, c_{e}\right\}$ is said eventually positive if $\exists d \geq 0$ such that $\left(A_{e}+d I\right) \stackrel{\vee}{>} 0$ and $\exists$ an $A_{e}$-invariant cone $\mathscr{K}_{e}$ for which $A_{e}$ is $\mathscr{K}_{e}$-positive and such that $b_{e} \in \mathscr{K}_{e}, c_{e} \in \mathscr{K}_{e}^{*}$.

Notice that $\mathscr{K}_{e}$ is $A_{e}$-invariant if and only if it is $A_{e}+d I$ invariant, and that $A_{e}$ is $\mathscr{K}_{e}$-positive if and only if $A_{e}+d I$ is $\mathscr{K}_{e}$-positive for $d \geq 0$. Armed with this definition we can obtain the continuous-time analogue of Theorem 6 .

Theorem 10 Consider a strictly proper rational transfer function $H(s)$ obeying Assumption 2. If $H(s)$ admits a minimal eventually positive realization then it is externally positive. Conversely, if $H(s)$ is externally positive and $\exists d \geq 0$ such that for $H_{d}(s-d)$ Algorithm 1 terminates successfully, then $H(s)$ has a minimal eventually positive realization.

Proof. Consider an eventually positive realization and the corresponding cone $\mathscr{K}_{e}$. Since $b_{e} \in \mathscr{K}_{e}$ and $c_{e} \in \mathscr{K}_{e}^{*}$, it is $c_{e} b_{e} \geq 0$. Since $A_{e}$-invariance implies $A_{e}+d I$-invariance of $\mathscr{K}_{e}$, then $\left(A_{e}+d I\right)^{k} b_{e} \in \mathscr{K}_{e} \forall k=1,2, \ldots, \forall d \geq 0$. Hence $c_{e}\left(A_{e}+d I\right)^{k} b_{e} \geq 0 \forall k=0,1,2, \ldots$, i.e., $H_{d}(s-d)$ is externally positive and so is $H(s)$. Conversely, if Algorithm 1 is successful for $H_{d}(s-d)$, then the triplet $\left\{A_{e}+d I, b_{e}, c_{e}\right\}$ is such that $\left(A_{e}+d I\right) \stackrel{\vee}{>} 0$ and Proposition 1 holds for it. Since the corresponding cone $\mathscr{K}_{e}$ is also $A_{e}$-invariant, $\left\{A_{e}, b_{e}, c_{e}\right\}$ is an eventually positive realization of $H(s)$. Minimality also follows from Proposition 1.

\subsection{A Nyquist-Shannon theorem for positivity of sampled systems}

Recall that for a continuous-time linear system

$$
\begin{aligned}
& \dot{x}=A x+b u \\
& y=c x,
\end{aligned}
$$

the $\mathrm{ZOH}$ discretization is given by

$$
\begin{aligned}
x(k+1) & =A_{\delta} x(k)+b_{\delta} u(k) \\
y(k) & =c_{\delta} x(k),
\end{aligned}
$$

where

$$
\begin{aligned}
A_{\delta} & =e^{A T} \\
b_{\delta} & =\int_{0}^{T} e^{A \tau} b d \tau \\
c_{\delta} & =c .
\end{aligned}
$$

The following theorem says that whenever a continuous-time system has an eventually positive realization, then provided the sampling time is sufficiently long, its $\mathrm{ZOH}$ discretization will be positive.

Theorem 11 Consider a strictly proper rational transfer function $H(s)$ satisfying Assumption 2. Assume $\exists r \in \mathbb{N}$, $r \neq 0$, such that $r \operatorname{Im}\left[\lambda_{i}-\lambda_{j}\right] \neq 2 \pi \xi, \xi= \pm 1, \pm 2, \ldots$, for all distinct poles $\lambda_{i}$ and $\lambda_{j}$ of $H(s)$ having $\operatorname{Re}\left[\lambda_{i}-\lambda_{j}\right]=$ 0 . If $H(s)$ admits a minimal eventually positive realization $\left\{A_{e}, b_{e}, c_{e}\right\}$ such that $b_{e} \geq 0$ and $c_{e} \geq 0$ and $\mathscr{R}_{e} \subset \mathbb{R}_{+}^{n}$, then $\exists T_{o}$ such that $\forall$ sample times $T \geq T_{o}$ the $\mathrm{ZOH}$ sampled realization $\left\{A_{\delta}, b_{\delta}, c_{\delta}\right\}$ obtained from it is minimal and positive.

Proof. $\left\{A_{e}, b_{e}, c_{e}\right\}$ eventually positive realization of $H(s)$ means that $\exists d \geq 0$ such that $\left(A_{e}+d I\right) \stackrel{\vee}{>} 0$, hence from Theorem $2 A_{e}$ is eventually exponentially positive, i.e., $\exists t_{o} \geq 0$ 
such that $e^{A_{e} t}>0 \forall t \geq t_{o}$. Consequently in (15) $A_{\delta}>0$ if $T \geq t_{o}$. In addition, from $e^{A_{e} \tau} b_{e}=\sum_{j=0}^{\infty} \frac{A_{e}^{j} \tau^{j}}{j !} b_{e}$, if $\mathscr{R}_{e} \subset \mathbb{R}_{+}^{n}$ then $e^{A_{e} \tau} b_{e} \geq 0 \forall \tau \geq 0$, meaning that also $b_{\delta} \geq 0$. Therefore $\left\{A_{\delta}, b_{\delta}, c_{\delta}\right\}$ is a positive realization of the $\overline{\mathrm{ZOH}}$ sampled system (14) when $T \geq t_{o}$. Analogously to the proof of Theorem 7 , the condition on $r$ prevents losses of controllability/observability due to merging of eigenvalues with identical real part. Hence $\exists T_{o} \geq t_{o}$ such that when the sampling time is $T \geq T_{o}$, the realization $\left\{A_{\delta}, b_{\delta}, c_{\delta}\right\}$ is also minimal.

Recall that when sampling a continuous-time linear system, if $\omega_{s}=\frac{2 \pi}{T}$ is the sampling frequency, then the Nyquist frequency $\omega_{N}=\frac{\omega_{s}}{2}=\frac{\pi}{T}$ delimits the spectral bandwidth of the output that is univocally represented in the sampled signal. The Nyquist sampling theorem is normally invoked to determine a $\omega_{N}$ sufficiently high, so that the interesting frequencies can be correctly reconstructed from the sampled signal.

The result in Theorem 11 can be interpreted as a "dual" to the Nyquist sampling theorem: provided $\omega_{N}$ is chosen sufficiently small (i.e., from Theorem $11, \omega_{N}<\frac{2 \pi}{T_{0}}$ ), it is possible to guarantee the existence of minimal discrete-time realizations whose internal state remains positive for all inputs and for all initial conditions. For externally positive systems, eigenvalue dominance conditions such as Assumption 2 imply that the system shows at most damped oscillations, and a high sampling period relies on this fact to eliminate undesired transients, like those violating positivity.

Example 4 Consider the following transfer function

$$
H(s)=\frac{8.843 s^{2}+11.86 s+8.95}{s^{3}+1.361 s^{2}+0.9938 s} .
$$

Since the poles are $\lambda=\{0,-0.6803 \pm 0.7286 i\}, H(s)$ satisfies Assumption 2. The impulse response of $H(s)$, shown in Fig. 4, implies that $H(s)$ is externally positive. Existence of a minimal positive realization is hard to verify even for such a simple system. The following is a minimal eventually positive realization

$$
\begin{gathered}
A_{e}=\left[\begin{array}{ccc}
-0.3513 & -0.6949 & 0.3599 \\
0.8229 & -0.8314 & 0.2594 \\
-0.0797 & 0.4214 & -0.1780
\end{array}\right], \\
b_{e}=\left[\begin{array}{c}
3.3472 \\
4.6663 \\
10.1462
\end{array}\right], \quad c_{e}=\left[\begin{array}{lll}
0.1621 & 0.0272 & 0.8056
\end{array}\right],
\end{gathered}
$$

for which $d=0.9889$. The effect of the damped oscillatory modes is to induce a transient violation of internal positivity, see Fig. 4. Since $t_{o}=4.84$, choosing a sample time $T=5$ one gets the positive $\mathrm{ZOH}$ minimal realization

$A_{\delta}=\left[\begin{array}{lll}0.0041 & 0.0447 & 0.1170 \\ 0.1140 & 0.0699 & 0.4012 \\ 0.2965 & 0.2044 & 0.8673\end{array}\right], b_{\delta}=\left[\begin{array}{c}9.2081 \\ 25.4148 \\ 52.6768\end{array}\right], c_{\delta}=c_{e}$

whose state and output impulse response is shown in Fig. 4 (blue and red curves). In this case, the sampling limits the bandwidth of the system without altering significantly the Bode plot for the amplitudes, see Fig 5. As a consequence of the long sampling time needed to have positivity, continuous-time and discrete-time output trajectories differ considerably.

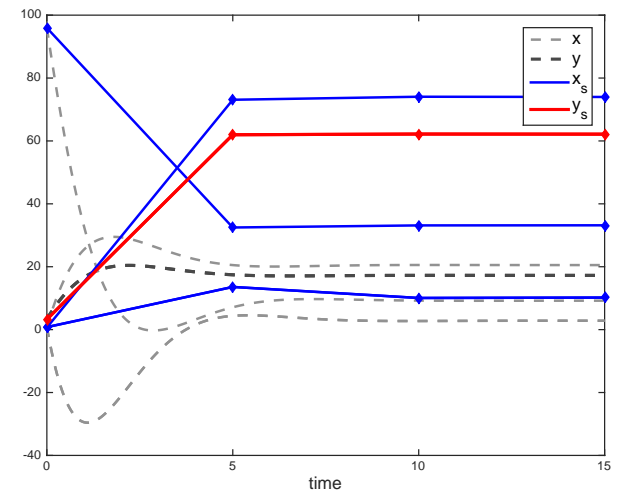

Fig. 4. Example 4. Impulse response of the continuous-time system (black, with state variables in gray) and of the $\mathrm{ZOH}$ sampled system (red, with state variables in blue). The continuous-time state violates positivity, while the $\mathrm{ZOH}$ state does not.

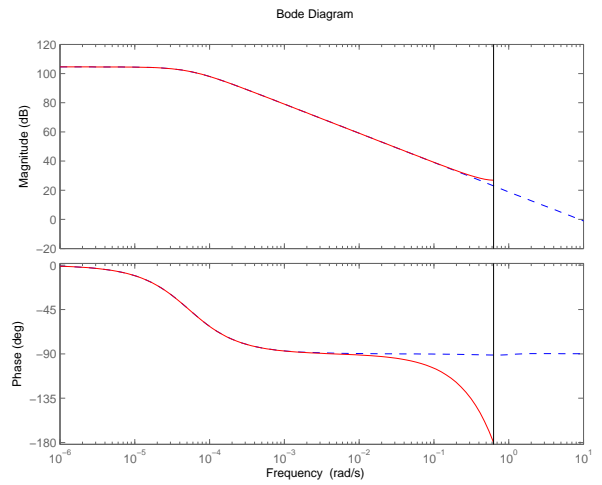

Fig. 5. Example 4. Bode plot of continuous-time (blue) and ZOH sampled transfer function (red). The vertical line represents the Nyquist frequency $\omega_{N}=\frac{\pi}{5}$.

\section{Conclusion}

In an attempt to fill the gap between externally and internally positive linear systems, a novel class of realizations in introduced in this paper. These correspond to state update matrices that are eventually positive, i.e., that become positive after a certain power. The constructive procedure we 
provide in the paper to obtain such minimal eventually positive realizations is easy and quite general and, in our experience, always terminating with success when applied to externally positive systems having a single strictly dominating real eigenvalue of multiplicity 1 . When the multiplicity is higher or when dominance is not strict, then the situation is more intricate and it is not clear whether eventual positivity can still play a key role. When the method is applicable, the insight into the structure of the systems that one gets through eventually positive realizations is considerable, as seen for example in our downsampling (or sampling, in continuous-time) theorems.

\section{Acknowledgements}

The author would like to thank Christian Grussler for discussions on the topic of the paper.

\section{References}

Altafini, C. 2015. Representing externally positive systems through minimal eventually positive realizations. In: 54 th IEEE Conference on Decision and Control.

Altafini, C., \& Lini, G. 2015. Predictable Dynamics of Opinion Forming for Networks With Antagonistic Interactions. Automatic Control, IEEE Transactions on, 60(2), 342-357.

Anderson, B.D.O., Deistler, M., Farina, L., \& Benvenuti, L. 1996. Nonnegative realization of a linear system with nonnegative impulse response. Circuits and Systems I: Fundamental Theory and Applications, IEEE Transactions on, 43(2), 134-142.

Benvenuti, L. 2013. Minimal Positive Realizations of Transfer Functions With Real Poles. Automatic Control, IEEE Transactions on, 58(4), 1013-1017.

Benvenuti, L., \& Farina, L. 2004. A tutorial on the positive realization problem. Automatic Control, IEEE Transactions on, 49(5), 651-664.

Benvenuti, L., Farina, L., Anderson, B.D.O., \& De Bruyne, F. 2000. Minimal positive realizations of transfer functions with positive real poles. Circuits and Systems I: Fundamental Theory and Applications, IEEE Transactions on, 47(9), 1370-1377.

Berman, A., \& Plemmons, R.J. 1994. Nonnegative matrices in the mathematical sciences. Classics in applied mathematics. Society for Industrial and Applied Mathematics.

Canto, R., Ricarte, B., \& Urbano, A.M. 2007. Positive Realizations of Transfer Matrices With Real Poles. Circuits and Systems II: Express Briefs, IEEE Transactions on, 54(6), 517-521.

Elhashash, Abed, \& Szyld, Daniel B. 2008. On general matrices having the Perron-Frobenius property. ELA. The Electronic Journal of Linear Algebra [electronic only], 17, 389-413.

Farina, L., \& Benvenuti, L. 1995. Positive realizations of linear systems. Systems \& Control Letters, 26(1), $1-9$.
Farina, L., \& Rinaldi, S. 2000. Positive Linear Systems: Theory and Applications. A Wiley-Interscience publication. Wiley.

Friedland, Shmuel. 1978. On an inverse problem for nonnegative and eventually nonnegative matrices. Israel Journal of Mathematics, 29(1), 43-60.

Guidorzi, R. 2014. Quasi-positive realization of externally positive discrete systems. Pages 43-48 of: Control Conference (ECC), 2014 European.

Hadjicostis, Christoforos. 1999. Bounds on the size of minimal nonnegative realizations for discrete-time $\{\mathrm{LTI}\}$ systems. Systems \& Control Letters, 37(1), 39 - 43.

Johnson, Charles R., \& Tarazaga, Pablo. 2004. On Matrices with Perron-Frobenius Properties and Some Negative Entries. Positivity, 8(4), 327-338.

Kim, Kyungsup. 2012. A construction method for positive realizations with an order bound. Systems \& Control Letters, 61(7), $759-765$.

Maeda, H., \& Kodama, S. 1981. Positive realization of difference equations. Circuits and Systems, IEEE Transactions on, 28(1), 39-47.

Nagy, B., \& Matolcsi, M. 2003. A lowerbound on the dimension of positive realizations. Circuits and Systems I: Fundamental Theory and Applications, IEEE Transactions on, 50(6), 782-784.

Nagy, B., \& Matolcsi, M. 2005. Minimal positive realizations of transfer functions with nonnegative multiple poles. Automatic Control, IEEE Transactions on, 50(9), 1447-1450.

Noutsos, D., \& Tsatsomeros, M. 2008. Reachability and Holdability of Nonnegative States. SIAM Journal on Matrix Analysis and Applications, 30(2), 700-712.

Noutsos, Dimitrios. 2006. On Perron-Frobenius property of matrices having some negative entries. Linear Algebra and its Applications, 412, 132 - 153.

Ohta, Yoshito, Maeda, Hajime, \& Kodama, Shinzo. 1984. Reachability, Observability, and Realizability of Continuous-Time Positive Systems. SIAM Journal on Control and Optimization, 22(2), 171-180.

Olesky, Dale D., Tsatsomeros, Michael J., \& van den Driessche, Pauline. 2009. $\mathrm{M}_{\vee}$-matrices: a generalization of $\mathrm{M}$ matrices based on eventually nonnegative matrices. ELA. The Electronic Journal of Linear Algebra, 18, 339-351.

Valcher, M., \& Farina, L. 2000. An Algebraic Approach to the Construction of Polyhedral Invariant Cones. SIAM Journal on Matrix Analysis and Applications, 22(2), 453471. 